\title{
CONSTRUCTION OF NONRECIPROCAL FUZZY PREFERENCE RELATIONS WITH THE USE OF PREFERENCE FUNCTIONS
}

\author{
Roberta Parreiras* and Petr Ekel
}

Received October 22, 2010 / Accepted December 12, 2011

\begin{abstract}
In order to model the preferences of a decision-maker (DM) by means of fuzzy preference relations, a DM can utilize different preference formats (such as ordering of the alternatives, utility values, multiplicative preference relations, fuzzy estimates, and reciprocal as well as nonreciprocal fuzzy preference relations) to express his/her judgments. Afterward, the obtained information is utilized to construct fuzzy preference relations. Here we introduce a procedure that allows the use of so-called preference functions (which is a preference format utilized in the methods of PROMETHEE family) to construct nonreciprocal fuzzy preference relations. With diverse preference formats being offered, a DM can select the one that is the most convenient to articulate his/her preferences. In order to demonstrate the applicability of the proposed procedure a multicriteria decision-making problem related to the site selection for constructing a new hospital is considered here.
\end{abstract}

Keywords: multicriteria decision-making, fuzzy preference relations, preference functions.

\section{INTRODUCTION}

The approaches to dealing with multicriteria (multiattribute) decision-making problems, which, for instance, are discussed in Orlovsky (1978), Chiclana et al. (1998), Ekel et al. (1998), Chiclana et al. (2001), are directed at processing the individual preferences as a pair $\langle X, R\rangle, X=$ $\left\{X_{1}, X_{2}, \ldots, X_{n}\right\}$ represents a finite and discrete set of alternatives, which are to be evaluated, compared, ordered, and/or prioritized under the consideration of a set of fuzzy nonstrict preference relations $R=\left\{R_{1}, R_{2}, \ldots, R_{q}\right\}$, given in accordance with a set of criteria $F=$ $\left\{F_{1}, F_{2}, \ldots, F_{q}\right\}$ being considered.

A fuzzy nonstrict preference relation (Orlovsky, 1978) consists of a binary fuzzy relation (BFR), which is a fuzzy set with bi-dimensional membership function $R_{p}\left(X_{k}, X_{l}\right): X \times X \rightarrow[0,1]$. In essence, the membership function of the $p$ th fuzzy preference relation indicates in the unit interval the degree to which the alternative $X_{k}$ is at least as good as $X_{l}$, when the criterion $F_{p}$,

*Corresponding author

Post-Graduate Program in Electrical Engineering, Pontifical Catholic University of Minas Gerais, Brazil.

E-mails: rop.asotech@gmail.com; pekel@superig.com.br 
is considered by a particular decision-maker (DM). In a somewhat loose sense $R_{p}\left(X_{k}, X_{l}\right)$ also represents the degree of truth of the statement " $X_{k}$ is at least as good as $X_{l}$ " (Kulshreshtha \& Shekar, 2000).

In dealing with $\langle X, R\rangle$ models, a fundamental question arises on how one can construct fuzzy preference relations to reflect the preferences of a DM. In practice, a DM can use different formats to establish preferences for the alternatives under consideration (Herrera-Viedma et al., 2002; Zhang et al., 2007; Pedrycz et al., 2011), including, the ordering of the alternatives, the utility values, multiplicative preference relations, fuzzy estimates, and fuzzy preference relations (additive reciprocal and nonreciprocal). In real world applications, several factors may lead a DM to select a different way for expressing his/her preferences about each criterion. Among these factors we can list the following (Pedrycz et al., 2011):

- each criterion comes with its significance (a fundamental feature which provides significance to the difference between two degrees evaluated on this criterion). Depending whether this significance has a qualitative or quantitative character, the use of certain preference formats can make the preference elicitation process easier and also more reliable;

- each criterion is associated with information arising from different sources and with information having different levels of uncertainty;

- a DM may find that his/her preference strengths can be better reflected or quantified by a specific preference format;

- a fact that a DM may posses previous knowledge or experience in expressing a specific preference format can motivate him/her to choose using it again.

The information captured by those different preference formats can be utilized to construct fuzzy preference relations by means of applying adequate transformation functions (Chiclana et al., 1998, 2001; Herrera-Viedma et al., 2002; Pedrycz et al., 2011). Alternatively, a DM can directly assess the fuzzy nonstrict preference relation by determining the preference strength of one alternative over another as a number in the unit interval. Current literature contains different encoding schemes which can be utilized in the construction of fuzzy preference relations (refer to (Wang, 1997) for instances of fuzzy preference relations based on different encoding schemes). In this paper, we consider a particular encoding scheme which can be utilized to construct a nonreciprocal fuzzy preference relation (NRFPR) (Orlovsky, 1978; Fodor \& Roubens, 1994a; Ekel \& Schuffner Neto, 2006; Pedrycz et al., 2011). Such encoding scheme allows one to construct a NRFPR which is compatible with:

- a rational approach (Ekel et al., 1998) for deriving fuzzy preference relations, which is based on the comparison of the fuzzy estimates provided by a DM to evaluate each alternative (a discussion of the advantages of such rational approach can be found in (Pedrycz et al., 2011));

- the notion of fuzzy nonstrict preference relation belonging to fuzzy preference structures with incomparability relation (Fodor \& Roubens, 1994a). In this way, the use of a NRFPR 
permits one to capture the preference degree of each pairwise comparison and the confidence degree of each pairwise comparison as well. A DM should assign a low credibility degree to a paired comparison whenever he/she can not define with conviction the preference degree for one alternative over another. The lack of conviction may be associated with missing information or to the occurrence of contradictory information about one alternative or both alternatives belonging to the pair (Fodor \& Roubens, 1994a,b).

Motivated by a demand for different preference formats to choose it as comfortable as possible for a DM to articulate his/her preferences (Zhang et al., 2007; Pedrycz et al., 2011), here we introduce a procedure that allows the use of preference functions to construct NRFPRs. In the methods of PROMETHEE family (Brans \& Vincke, 1985), a DM must define the shape of the preference functions in accordance with his/her preferences so that they can be utilized to compare the alternatives and to construct outranking relations for each criterion being considered in the multicriteria analysis. Here, the preference functions are implemented as part of a general framework for preference modeling in a fuzzy environment, which offers six preference formats for preference articulation. They are: the ordering of the alternatives, the utility values, the multiplicative preference relations, the fuzzy estimates, the fuzzy preference relations (reciprocal and nonreciprocal), and the preference functions. With the availability of several preference formats, the choice of the most suitable one is a prerogative of a DM. The applicability of the preference functions to construct NRFPRs, within such general framework for preference modeling, is demonstrated through the solution of a decision-making problem related to the site selection for constructing a new hospital (Vahidnia et al., 2009).

\section{JUDGMENTS OF STRICT PREFERENCE, INDIFFERENCE, AND INCOMPARABILITY}

Let us consider that a DM is asked to compare two alternatives $X_{k} \in X, X_{l} \in X$ for a given criterion and determine which, between these two, he/she prefers. One of the following answers is expected (Fodor \& Roubens, 1994a):

- $X_{k}$ and $X_{l}$ are indifferent;

- $X_{k}$ is strictly better than $X_{l}$;

- $X_{l}$ is strictly better than $X_{k}$;

- $X_{k}$ and $X_{l}$ are incomparable (a DM may not be able to compare the alternatives due to missing or uncertain information or as a consequence of the existence of conflicting information).

Accordingly, in order to realistically characterize this comparison between two alternatives, three main types of judgments can be distinguished, namely indifference, strict preference, and incomparability. Given the $p$ th criterion, these judgments can be modeled by means of three specific BFRs, namely the fuzzy indifference relation $I_{p}$, the fuzzy strict preference relation $P_{p}$, and the fuzzy incomparability relation $J_{p}$, in such a way that the membership function of each BFR quantifies in the interval $[0,1]$, the credibility or the intensity of the observed judgment. 
In fuzzy preference models based on BFRs, given De Morgan triplet $(T, S, N)$, it is possible to define $I_{p}, P_{p}$, and $J_{p}$ exclusively in terms of the fuzzy nonstrict preference relation $R_{p}$. It is important to indicate that the fuzzy nonstrict preference relation, by definition, can be expressed as (Fodor \& Roubens, 1994a):

$$
R_{p}=P_{p} \cup I_{p}
$$

By considering that $I_{p}$ corresponds to all pairs of alternatives that simultaneously satisfy $R_{p}\left(X_{k}, X_{l}\right)$ and $R_{p}\left(X_{l}, X_{k}\right)$, the indifference relation can be stated as

$$
I_{p}=R_{p} \cap R_{p}^{-1}
$$

where $R_{p}^{-1}$ is the inverse relation of $R_{p}$, that is $R_{p}^{-1}\left(X_{k}, X_{l}\right)=R_{p}\left(X_{l}, X_{k}\right)$ (Orlovsky, 1978, 1981).

Similarly, as $P_{p}\left(X_{k}, X_{l}\right)$ implies that $R_{p}\left(X_{k}, X_{l}\right)$ and $N\left(R_{p}\left(X_{l}, X_{k}\right)\right)$, the strict preference can be specified as

$$
P_{p}=R_{p} \cap R_{p}^{d},
$$

where $R^{d}$ corresponds to the dual relation of $R_{p}$, that is

$$
\left.R_{p}^{d}\left(X_{k}, X_{l}\right)=1-R_{p}\left(X_{l}, X_{k}\right)\right)
$$

(Fodor \& Roubens, 1994a).

Finally, as the relation $J_{p}\left(X_{k}, X_{l}\right)$ implies that $N\left(R_{p}\left(X_{k}, X_{l}\right)\right)$ and $N\left(R_{p}\left(X_{l}, X_{k}\right)\right)$, the incomparability relation is given by

$$
J_{p}=\bar{R}_{p} \cap R_{p}^{d}
$$

where $\bar{R}_{p}$ corresponds to the complementary relation of $R_{p}$ that is

$$
\overline{R_{p}\left(X_{k}, X_{l}\right)}=1-R_{p}\left(X_{k}, X_{l}\right)
$$

(Fodor \& Roubens, 1994a).

Therefore, once we have at hand the values of $R_{p}\left(X_{k}, X_{l}\right)$ and $R_{p}\left(X_{l}, X_{k}\right)$, the estimation of $I_{p}$, $P_{p}$, and $J_{p}$ is realized on the basis of (2), (3), and (4), respectively. As one can note, those three expressions require the selection of a $t$-norm operator. Unfortunately, as it has been discussed, for instance, in De Baets \& Fodor (1997), it is not simple to select a $t$-norm to implement (2)-(4), if we want to preserve certain desirable properties of a fuzzy preference structure. Indeed, a negative result demonstrated in Alsina (1985) indicates that, if a De Morgan triplet is utilized to represent the complement, the intersection and the union of BFRs, then the equality

$$
Z=(Z \cap W) \cup(Z \cap \bar{W})
$$

is not satisfied for any binary fuzzy relations $Z$ and $W$. If we consider $Z$ as being $R_{p}$ and $W$ as $R_{p}^{d}$, then it implies that the relationships (2), (3), and (1) are inconsistent for any reflexive fuzzy binary relation, if we use the same intersection operator to implement the two intersection operations in (5). 
Among the admissible $t$-norms to be utilized in this context (Fodor \& Roubens, 1994b), we selected the min operator to implement the intersection in (2) and in (4) and the Lukasiewicz $t$-norm to implement the intersection in (3), as given by the following expressions:

$$
\begin{aligned}
& I_{p}\left(X_{k}, X_{l}\right)=\min \left\{R_{p}\left(X_{k}, X_{l}\right), R_{p}\left(X_{l}, X_{k}\right)\right\} \\
& P_{p}\left(X_{k}, X_{l}\right)=\min \left\{R_{p}\left(X_{k}, X_{l}\right)-R_{p}\left(X_{l}, X_{k}\right), 0\right\} \\
& J_{p}\left(X_{k}, X_{l}\right)=\min \left\{1-R_{p}\left(X_{k}, X_{l}\right), 1-R_{p}\left(X_{l}, X_{k}\right)\right\}
\end{aligned}
$$

It should be indicated that the definition of the fuzzy strict preference relation as given by (7) is in conformity with the Orlovsky choice procedure (Orlovsky, 1978), which is described in Section 5.

\section{DIRECT ASSESSMENT OF NONRECIPROCAL FUZZY NONSTRICT PREFERENCE RELATIONS}

In real applications, a DM is usually asked to pick up the values in the unit interval that reflect the level of credibility or just the strength of his/her nonstrict preference for one alternative over the other. The encoding scheme considered here is reflected by a NRFPR which verifies the following conditions (Pedrycz et al., 2011):

- if $R_{p}\left(X_{k}, X_{l}\right)=1$ and $R_{p}\left(X_{l}, X_{k}\right)=1$, then $X_{k}$ is indifferent to $X_{l}$;

- if $R_{p}\left(X_{k}, X_{l}\right)=1$ and $R_{p}\left(X_{l}, X_{k}\right)=0$, then $X_{k}$ is strictly preferred to $X_{l}$;

- if $R_{p}\left(X_{k}, X_{l}\right)=0$ and $R_{p}\left(X_{l}, X_{k}\right)=1$, then $X_{l}$ is strictly preferred to $X_{k}$;

- if $R_{p}\left(X_{k}, X_{l}\right)=0$ and $R_{p}\left(X_{l}, X_{k}\right)=0$, then $X_{k}$ and $X_{l}$ are not comparable;

- the entries of the main diagonal are filled with 1 , due to the reflexivity of $R_{p}\left(X_{k}, X_{l}\right)$.

Intermediate judgments among the situations described above are also allowed. They can be interpreted as follows:

- if $0<R_{p}\left(X_{k}, X_{l}\right)<1$ and $R_{p}\left(X_{l}, X_{k}\right)=1$, then $X_{l}$ is weakly preferred to $X_{k}$;

- if $R_{p}\left(X_{k}, X_{l}\right)=1$ and $0<R_{p}\left(X_{l}, X_{k}\right)<1$, then $X_{k}$ is weakly preferred to $X_{l}$;

- if $0<R_{p}\left(X_{k}, X_{l}\right)<1$ and $R_{p}\left(X_{l}, X_{k}\right)=0$, then $X_{k}$ is weakly preferred to $X_{l}$ and, at the same time, $X_{k}$ and $X_{l}$ are to a degree considered incomparable;

- if $R_{p}\left(X_{k}, X_{l}\right)=0$ and $0<R_{p}\left(X_{l}, X_{k}\right)<1$, then $X_{l}$ is weakly preferred to $X_{k}$ and, at the same time, $X_{k}$ and $X_{l}$ are to a degree considered incomparable. 
It is important to indicate that relations (6) and (7) determine a clear relationship between the values of $R_{p}\left(X_{k}, X_{l}\right)$ and $R_{p}\left(X_{l}, X_{k}\right)$. If (6) and (7) are utilized for defining the fuzzy indifference relation and the fuzzy strict preference relation, respectively, then the difference between $R_{p}\left(X_{k}, X_{l}\right)$ and $R_{p}\left(X_{l}, X_{k}\right)$ reflects the level of strict preference between the alternatives $X_{k}$ and $X_{l}$. Besides, the minimum value between $R_{p}\left(X_{k}, X_{l}\right)$ and $R_{p}\left(X_{l}, X_{k}\right)$ reflects the level of indifference between the alternatives $X_{k}$ and $X_{l}$.

With reference to the consistency of NRFPRs, the weak transitivity, which is given by (HerreraViedma et al., 2004).

If $R_{p}\left(X_{k}, X_{j}\right)>R_{p}\left(X_{j}, X_{k}\right)$ and $R_{p}\left(X_{j}, X_{l}\right)>R_{p}\left(X_{l}, X_{j}\right)$, then

$$
R_{p}\left(X_{k}, X_{l}\right)>R_{p}\left(X_{l}, X_{k}\right), \quad \forall X_{k}, X_{j}, X_{l} \in X
$$

leads to the minimum requirement that should be satisfied by rational preference judgments. It implies that if someone says that $X_{k}$ is preferred to $X_{j}$ and that $X_{j}$ is preferred to $X_{l}$, then $X_{k}$ is preferred to $X_{l}$, without considering the strength of the preferences. It is also the minimum requirement for the application of decision-making methods based on the Orlovsky choice function (Orlovsky, 1978), if one wants some rational properties to be attained (Sengupta, 1998).

\section{INDIRECT ASSESSMENT OF NONRECIPROCAL FUZZY NONSTRICT PREFERENCE RELATIONS}

In individual (as well as in group decision-making), when different preference formats are utilized by a DM to express his/her preferences, the information must be made uniform under adequate transformation functions, before being analyzed. These transformation functions can convert heterogeneous preference information, which may be qualitative or quantitative, two-valued or fuzzy, ordered or non-ordered, ordinal or cardinal into fuzzy preference relations, which form a more general preference model (Chiclana et al., 1998, 2001; Herrera-Viedma et al., 2002; Pedrycz et al., 2011). As it was indicated above, the five main formats, that can be utilize by a DM to articulate his/her preferences, in addition to the NRFPRs, are:

- Vector with the ordering of all alternatives. The ordering of alternatives from best to worst can be represented as an array $O_{p}=\left[O_{p}\left(X_{1}\right), \ldots, O_{p}\left(X_{n}\right)\right]$, with $O_{p}\left(X_{k}\right)$ being a permutation function, which returns the position of alternative $X_{k}$ among the integer values $\{1,2, \ldots, n\}$ (Chiclana et al., 1998).

- Vector of the utility values. A measurable utility function $U_{p}(x): X \rightarrow[0,1]$, founded on differences in preference strengths (Belton, 1999; Farquhar \& Keller, 1989), allows a DM to assign a utility value from the unity interval $[0,1]$ to each alternative belonging to $X$.

- Multiplicative preference relation. The multiplicative preference relation can be represented as a $n \times n$ positive reciprocal matrix $M_{p}$ reflecting the preference intensity ratio between the alternatives in accordance with the Analytic Hierarchy Process approach 
(Saaty, 1980). Each entry $M_{p}\left(X_{k}, X_{l}\right)$ of this reciprocal matrix represents a preference intensity ratio and can be interpreted as " $X_{k}$ is $M_{p}\left(X_{k}, X_{l}\right)$ times more dominant than $X_{l}$ " (Saaty, 1980) or as " $X_{k}$ is $M_{p}\left(X_{k}, X_{l}\right)$ times as good as $X_{l}$ " (Chiclana et al., 2001). Under the condition of multiplicative reciprocity, once a DM provides $M_{p}\left(X_{k}, X_{l}\right)$, the value of $M_{p}\left(X_{l}, X_{k}\right)$ is automatically inferred as $M_{p}\left(X_{l}, X_{k}\right)=1 / M_{p}\left(X_{k}, X_{l}\right)$.

- Fuzzy estimates. The elements of $X$ can be evaluated with the use of fuzzy estimates $L_{p}=\left\{l_{p}\left(X_{1}\right), \ldots, l_{p}\left(X_{n}\right)\right\}$, being $l_{p}\left(X_{k}\right)$ the fuzzy estimate associated with alternative $X_{k}$ from the point of view of a given criterion $F_{p}$. The fuzzy estimate $l_{p}\left(X_{k}\right)$ refers to a fuzzy number that can be directly specified by a DM or indirectly expressed by means of linguistic terms from some set $S$ such as, for instance, $S\left(F_{p}\right)=$ \{low quality, average quality, high quality\}. In the latter case, the linguistic terms must be converted into fuzzy estimates, as required to perform the analysis of the problem.

- Additive reciprocal fuzzy preference relation (ARFPR). An ARFPR consists of a specific type of fuzzy preference relation which satisfies the additive reciprocity condition:

$$
R R_{p}\left(X_{k}, X_{l}\right)+R R_{p}\left(X_{l}, X_{k}\right)=1, \quad \forall X_{k}, X_{l} \in X,
$$

where $R R_{p}$ represents the ARFPR (here, NRFPR is denoted as $R_{p}$ and ARFPR is denoted as $\left.R R_{p}\right)$.

The encoding scheme usually associated with ARFPRs can be summarized as the following rules:

- $R R_{p}\left(X_{k}, X_{l}\right)=0.5$ means that $X_{k}$ is indifferent to $X_{l}$;

- $0 \leq R R_{p}\left(X_{k}, X_{l}\right)<0.5$ means that $X_{l}$ is preferred to $X_{k}$;

- $0.5<R R\left(X_{k}, X_{l}\right) \leq 1$ means that $X_{k}$ is preferred to $X_{l}$;

- the entries of the main diagonal are filled with 0.5 , as each element is equal to itself and, as a result, indifferent to itself.

In (Chiclana et al., 1998), one can find different transformation functions for converting a vector with the ordering of all alternatives or a vector of the utility values associated with each alternative into an ARFPR. A transformation function for converting multiplicative preference relations into an ARFPR is proposed in (Chiclana et al., 2001). In (Pedrycz et al., 2011), new transformation functions are proposed. They convert preference information expressed in those five different formats described above into NRFPRs. Some of the transformation functions proposed in (Queiroz, 2009; Pedrycz et al., 2011) are summarized in Table 1. It should be indicated that they are defined for the case of maximization criteria (if the criterion being considered has a minimization character, then the direction of the signs of all the inequalities appearing in the transformation functions should be reversed). 
Table 1 - Transformation functions for the conversion of preference information expressed in different formats into a NRFPR (Pedrycz et al., 2011).

\begin{tabular}{|c|c|}
\hline Original preference format & Transformation function \\
\hline $\begin{array}{l}\text { Vector with the ordering } \\
\text { of all alternatives }\end{array}$ & $\begin{array}{l}R\left(X_{k}, X_{l}\right)=H_{1}\left(O\left(X_{k}\right), O\left(X_{l}\right)\right)= \\
\begin{cases}\frac{1}{2}+\frac{O\left(X_{l}\right)-O\left(X_{k}\right)}{2(n-1)}, & \text { if } O\left(X_{k}\right)>O\left(X_{l}\right) \\
1, & \text { if } O\left(X_{k}\right) \leq O\left(X_{l}\right)\end{cases} \end{array}$ \\
\hline $\begin{array}{l}\text { Vector of the utility values } \\
\text { based on an interval scale }\end{array}$ & $\begin{array}{l}R\left(X_{k}, X_{l}\right)=H_{2}\left(U\left(X_{k}\right), U\left(X_{l}\right)\right)= \\
\begin{cases}1+U\left(X_{k}\right)-U\left(X_{l}\right), & \text { if } U\left(X_{k}\right)<U\left(X_{l}\right) \\
1, & \text { if } U\left(X_{k}\right) \geq U\left(X_{l}\right)\end{cases} \end{array}$ \\
\hline $\begin{array}{l}\text { Multiplicative preference } \\
\text { relation }\end{array}$ & $\begin{array}{l}R\left(X_{k}, X_{l}\right)=H_{3}\left(M\left(X_{k}, X_{l}\right), M\left(X_{l}, X_{l}\right)\right)= \\
\begin{cases}1+\frac{1}{2} \log _{m} \frac{M\left(X_{k}, X_{l}\right)}{M\left(X_{l}, X_{k}\right)}, & \text { if } \log _{m} M\left(X_{k}, X_{l}\right)<0 \\
1, & \text { if } \log _{m} M\left(X_{k}, X_{l}\right) \geq 0\end{cases} \end{array}$ \\
\hline $\begin{array}{l}\text { Additive reciprocal fuzzy } \\
\text { preference relation }\end{array}$ & $\begin{array}{l}R\left(X_{k}, X_{l}\right)=H_{4}\left(R R\left(X_{k}, X_{l}\right), R R\left(X_{l}, X_{k}\right)\right)= \\
\begin{cases}1+R R\left(X_{k}, X_{l}\right)-R R\left(X_{l}, X_{k}\right), & \text { if } R R\left(X_{k}, X_{l}\right)<0.5 \\
1, & \text { if } R R\left(X_{k}, X_{l}\right) \geq 0.5\end{cases} \end{array}$ \\
\hline
\end{tabular}

In this paper, those transformation functions summarized in Table 1 are utilized to create a general framework for constructing NRFPRs on the basis of preference information which may be expressed in different formats. Given a set $X$ of alternatives, a DM can express his/her preferences by ordering the alternatives from best to worst, by constructing a utility function or by performing pairwise comparisons between the alternatives to construct a multiplicative preference relation, a ARFPR or a NRFPR. At the same time, in order to increase the flexibility of such general framework, the results of (Ekel et al., 1998) are utilized to derive NRFPRs from the fuzzy estimates given by a DM to evaluate the alternatives. In this way, once a DM provides fuzzy estimates $F_{p}\left(X_{k}\right), k=1,2, \ldots, n$ to evaluate all the alternatives for a criterion $F_{p}$ which can be measured on a numerical scale, if the essence of preference is coherent with the natural order $(\geq)$ along the axis of measurable values of $F_{p}$, then the following expressions can be utilized to construct a NRFPR:

$$
\begin{aligned}
& R_{p}\left(X_{k}, X_{l}\right)=\sup _{\substack{f_{p}\left(X_{k}\right), f_{p}\left(X_{l}\right) \in F_{p} \\
f_{p}\left(X_{k}\right) \geq f_{p}\left(X_{l}\right)}} \min \left(F_{p}\left(f_{p}\left(X_{k}\right)\right), F_{p}\left(f_{p}\left(X_{l}\right)\right)\right), \\
& R_{p}\left(X_{l}, X_{k}\right)=\sup _{\substack{f_{p}\left(X_{k}\right), f_{p}\left(X_{l}\right) \in F_{p} \\
f_{p}\left(X_{l}\right) \geq f_{p}\left(X_{k}\right)}} \min \left(F_{p}\left(f_{p}\left(X_{k}\right)\right), F_{p}\left(f_{p}\left(X_{l}\right)\right)\right),
\end{aligned}
$$

where $f_{p}\left(X_{k}\right)$ and $f_{p}\left(X_{l}\right)$ are real numbers reflecting the evaluation of the attribute $F_{p}$ (with a maximization character) for the alternatives $X_{k}$ and $X_{l} ; F_{p}\left(f_{p}\left(X_{k}\right)\right)$ and $F_{p}\left(f_{p}\left(X_{l}\right)\right)$ represent the membership functions of the fuzzy sets $F_{p}\left(X_{k}\right)$ and $F_{p}\left(X_{l}\right)$ evaluated at $f_{p}\left(X_{k}\right)$ and $f_{p}\left(X_{l}\right)$, respectively. It should be indicated that (11) and (12) are defined for maximization criteria. The direction of the signs of the inequalities in (11) and (12) should be reversed, if a minimization criterion is being considered. 


\section{CONSTRUCTION OF NONRECIPROCAL FUZZY PREFERENCE RELATIONS WITH THE USE OF PREFERENCE FUNCTIONS}

The results of (Brans \& Vincke, 1985) permit one to apply preference functions as an additional format that can be used by a DM to express his/her preferences. In particular, in the family of PROMETHEE methods, the preferences being restricted to a single criterion $F_{p}$, are modeled through a preference function $s_{p}\left(X_{k}, X_{l}\right)$, in such a way that it reflects the preference level of $X_{k}$ over $X_{l}$, according to the following rules:

- if $s_{p}\left(X_{k}, X_{l}\right)=0$, both alternatives are considered indifferent to each other;

- if $s_{p}\left(X_{k}, X_{l}\right)=1, X_{k}$ is strictly preferred to $X_{l}$;

- if $0<s_{p}\left(X_{k}, X_{l}\right)<1, X_{k}$ is weakly preferred to $X_{l}$.

The preference functions are usually defined in terms of the difference

$$
d_{p}\left(X_{k}, X_{l}\right)=f_{p}\left(X_{k}\right)-f_{p}\left(X_{l}\right)
$$

in such a way that they transform the difference in the evaluations of two alternatives into a preference intensity between 0 and 1 . The preference functions can have different shapes, as long as they are nondecreasing functions of the difference $d_{p}\left(X_{k}, X_{l}\right)$ (Brans \& Vincke, 1985). The methods PROMETHEE I and PROMETHEE II admit six different generalized models for the preference function, which cover most part of the scenarios encountered in real applications (Brans \& Vincke, 1985). They are: the usual criterion, the quasi-criterion, the level-criterion, the linear criterion, the linear criterion with indifference region, and the Gaussian criterion. With the availability of those generalized functions, it may be easier for a DM to define a preference function reflecting his/her preferences. Next, we describe how one can construct NRFPRs based on each one of those preference functions. It should be indicated that, here we consider the criteria as being of maximization type (without loss of generality).

Let us begin by considering three preference functions that are particularly easy to define: the usual criterion, the quasi-criterion, and the level-criterion. Figure 1 shows the graphical representation of the usual criterion, which may be considered as being the simplest type of preference function, since it does not require any parameter to be set by a DM. As it can be seen in Figure 1 , the level of strict preference of $X_{k}$ over $X_{l}$ is equal to 1 for $d_{p}\left(X_{k}, X_{l}\right)>0$ and is null for $d_{p}\left(X_{k}, X_{l}\right)<0$. If a DM wants to construct a NRFPR based on the usual criterion, the following rules must be applied to the comparison of each pair of alternatives:

- if $d_{p}\left(X_{k}, X_{l}\right)<0$, then $R_{p}\left(X_{k}, X_{l}\right)=0$ and $R_{p}\left(X_{l}, X_{k}\right)=1$;

- if $d_{p}\left(X_{k}, X_{l}\right)=0$, then $R_{p}\left(X_{k}, X_{l}\right)=1$ and $R_{p}\left(X_{l}, X_{k}\right)=1$;

- if $d_{p}\left(X_{k}, X_{l}\right)>0$, then $R_{p}\left(X_{k}, X_{l}\right)=1$ and $R_{p}\left(X_{l}, X_{k}\right)=0$. 

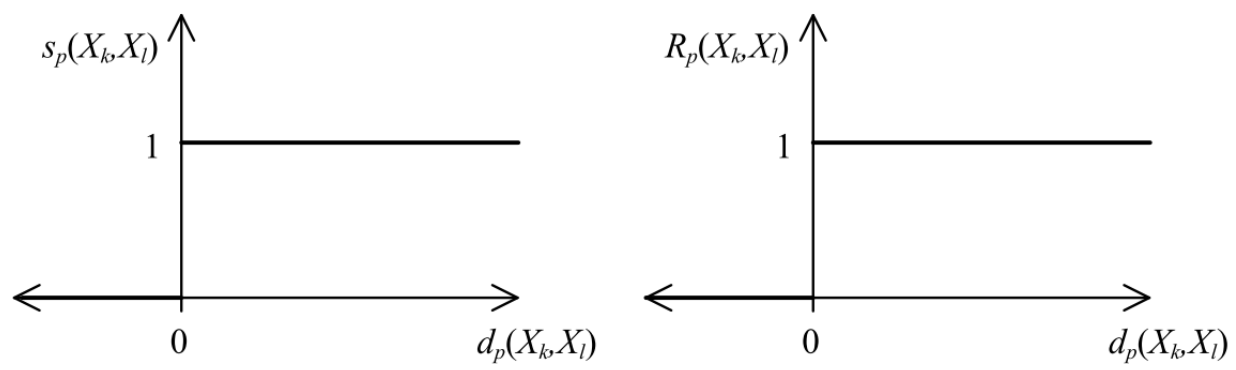

Figure 1 - Left side: Preference function based on the usual criterion; Right side: NRFPR based on the usual criterion, expressed in function of the difference $d_{p}\left(X_{k}, X_{l}\right)$.

Figure 2 shows a graphical representation of the preference function corresponding to the quasicriterion and of the NRFPR based on the quasi-criterion. It is worth noting that the usual criterion can be seen as a particular case of the quasi-criterion where the bounds of the indifference interval [ $\left.-a_{p}, a_{p}\right]$ are null, that is $a_{p}=0$. If a DM wants to construct a NRFPR coherently with the quasi-criterion, the pairwise comparisons must attain the following conditions:

- if $d_{p}\left(X_{k}, X_{l}\right)<-a_{p}$, then $R_{p}\left(X_{k}, X_{l}\right)=0$ and $R_{p}\left(X_{l}, X_{k}\right)=1$;

- if $-a_{p} \leq d_{p}\left(X_{k}, X_{l}\right) \leq a_{p}$, then $R_{p}\left(X_{k}, X_{l}\right)=1$ and $R_{p}\left(X_{l}, X_{k}\right)=1$;

- if $d_{p}\left(X_{k}, X_{l}\right)>a_{p}$, then $R_{p}\left(X_{k}, X_{l}\right)=1$ and $R_{p}\left(X_{l}, X_{k}\right)=0$.
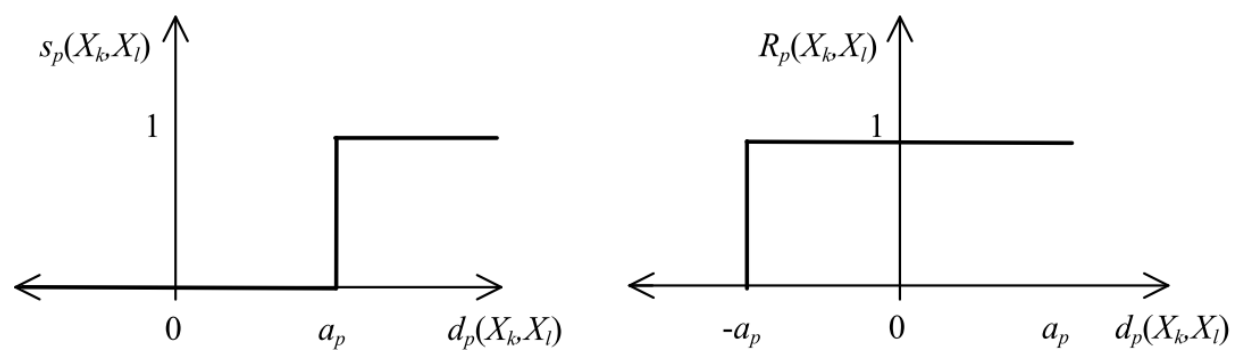

Figure 2 - Left side: Preference function based on the quasi-criterion; Right side: NRFPR based on the quasi-criterion, expressed in function of the difference $d_{p}\left(X_{k}, X_{l}\right)$.

The level-criterion allows discriminating an indifference interval $\left[-a_{p}, a_{p}\right]$ and a weak preference interval $\left[a_{p}, b_{p}\right]$ (and $\left[-b_{p},-a_{p}\right]$ ). The pairwise comparisons must satisfy the following conditions in order to construct a NRFPR coherently with the level-criterion:

- if $d_{p}\left(X_{k}, X_{l}\right) \leq-b_{p}$, then $R_{p}\left(X_{k}, X_{l}\right)=0$ and $R_{p}\left(X_{l}, X_{k}\right)=1$;

- if $-b_{p}<d_{p}\left(X_{k}, X_{l}\right) \leq-a_{p}$, then $R_{p}\left(X_{k}, X_{l}\right)=0.5$ and $R_{p}\left(X_{l}, X_{k}\right)=1$;

- if $-a_{p}<d_{p}\left(X_{k}, X_{l}\right)<a_{p}$, then $R_{p}\left(X_{k}, X_{l}\right)=1$ and $R_{p}\left(X_{l}, X_{k}\right)=1$;

- if $a_{p}<d_{p}\left(X_{k}, X_{l}\right)<b_{p}$, then $R_{p}\left(X_{k}, X_{l}\right)=1$ and $R_{p}\left(X_{l}, X_{k}\right)=0.5$;

- if $d_{p}\left(X_{k}, X_{l}\right) \geq b_{p}$, then $R_{p}\left(X_{k}, X_{l}\right)=1$ and $R_{p}\left(X_{l}, X_{k}\right)=0$. 

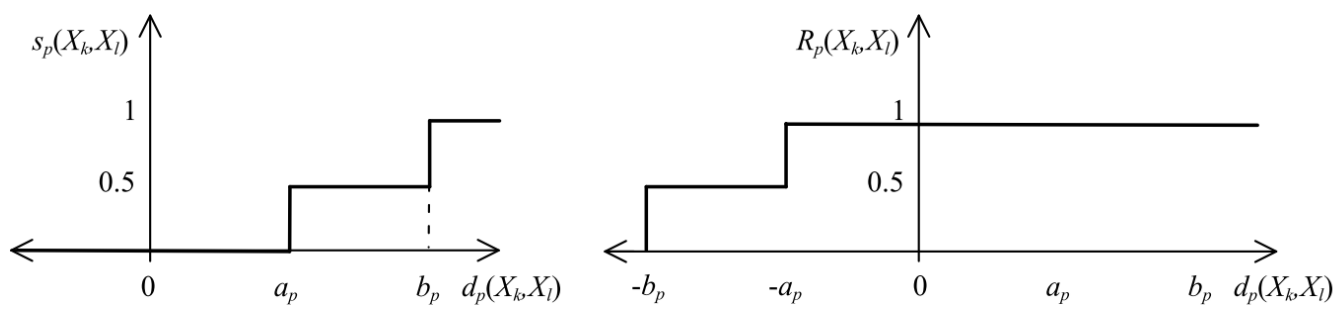

Figure 3 - Left side: Preference function based on the level criterion; Right side: NRFPR based on the level criterion, expressed in function of the difference $d_{p}\left(X_{k}, X_{l}\right)$.

The preference functions of the linear criterion, the linear criterion with an indifference region, and the Gaussian criterion present a smooth transition between indifference and strict preference, which permits a DM to make judgments at different levels of weak preference. The graphical representations of those preference functions are shown in the Figures 4, 5, and 6, respectively.

In the visualization of the linear criterion (refer to Fig. 4), the slope of the preference function depend on the value of the preference threshold $b_{p}$. Thus, in order to define a NRFPR coherently with the linear criterion, the pairwise comparisons must be in accordance with the following conditions:

- if $d_{p}\left(X_{k}, X_{l}\right)<-b_{p}$, then $R_{p}\left(X_{k}, X_{l}\right)=0$ and $R_{p}\left(X_{l}, X_{k}\right)=1$;

- if $-b_{p} \leq d_{p}\left(X_{k}, X_{l}\right)<0$, then $R_{p}\left(X_{k}, X_{l}\right)=1-\left|d_{p}\left(X_{k}, X_{l}\right)\right| / b_{p}$ and $R_{p}\left(X_{l}, X_{k}\right)=1$;

- if $0 \leq d_{p}\left(X_{k}, X_{l}\right)<-b_{p}$, then $R_{p}\left(X_{k}, X_{l}\right)=1$ and $R_{p}\left(X_{l}, X_{k}\right)=1-\left|d_{p}\left(X_{k}, X_{l}\right)\right| / b_{p}$;

- if $d_{p}\left(X_{k}, X_{l}\right)>b_{p}$, then $R_{p}\left(X_{k}, X_{l}\right)=1$ and $R_{p}\left(X_{l}, X_{k}\right)=0$.
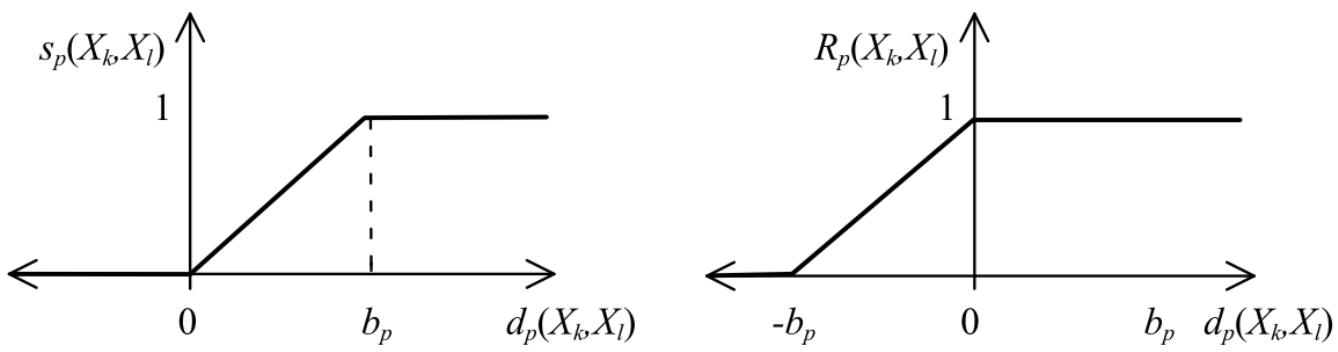

Figure 4 - Left side: Preference function based on the linear criterion; Right side: NRFPR based on the linear criterion, expressed in function of the difference $d_{p}\left(X_{k}, X_{l}\right)$.

The Linear criterion with indifference region can be considered an extension of the linear criterion to deal with judgments of indifference when the pair of alternatives have similar evaluations and their difference lies in an indifference interval $\left[-a_{p}, a_{p}\right]$. A NRFPR is coherent with this definition of linear criterion with indifference region if it is in accordance with the following conditions (refer to Fig. 5): 
- if $d_{p}\left(X_{k}, X_{l}\right)<-b_{p}$, then $R_{p}\left(X_{k}, X_{l}\right)=0$ and $R_{p}\left(X_{l}, X_{k}\right)=1$;

- if $-b_{p} \leq d_{p}\left(X_{k}, X_{l}\right)<-a_{p}$, then $R_{p}\left(X_{k}, X_{l}\right)=1-\left|d_{p}\left(X_{k}, X_{l}\right)\right| / b_{p}$ and $R_{p}\left(X_{l}, X_{k}\right)=1$;

- if $-a_{p} \leq d_{p}\left(X_{k}, X_{l}\right) \leq a_{p}$, then $R_{p}\left(X_{k}, X_{l}\right)=1$ and $R_{p}\left(X_{l}, X_{k}\right)=1$;

- if $a_{p}<d_{p}\left(X_{k}, X_{l}\right) \leq b_{p}$, then $R_{p}\left(X_{k}, X_{l}\right)=1$ and $R_{p}\left(X_{l}, X_{k}\right)=1-\left|d_{p}\left(X_{k}, X_{l}\right)\right| / b_{p}$;

- if $d_{p}\left(X_{k}, X_{l}\right)>b_{p}$, then $R_{p}\left(X_{k}, X_{l}\right)=1$ and $R_{p}\left(X_{l}, X_{k}\right)=0$.
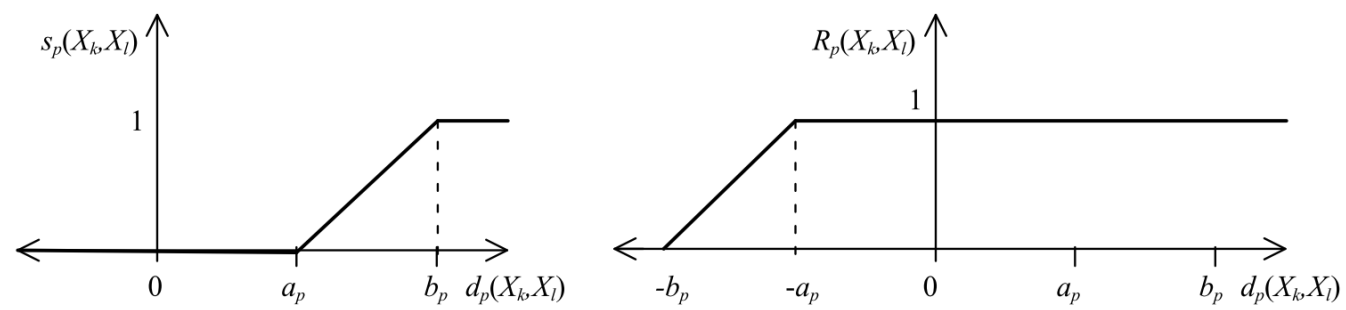

Figure 5 - Left side: Preference function based on the linear criterion with indifference region; Right side: NRFPR based on the linear criterion with indifference region, expressed in function of the difference $d_{p}\left(X_{k}, X_{l}\right)$.

Finally, in the case of the Gaussian criterion, $\sigma_{p}$ is the distance between the origin and the inflexion point of the curve $s_{p}\left(X_{k}, X_{l}\right)$. The Gaussian Criterion allows a transition (without discontinuities) between judgments of indifference and strict preference. In order to define a NRFPR coherently with the Gaussian criterion, the pairwise comparisons must be in accordance with the following conditions:

- if $d_{p}\left(X_{k}, X_{l}\right)<0$, then $R_{p}\left(X_{k}, X_{l}\right)=\exp \left(-\left|d_{p}\left(X_{k}, X_{l}\right)\right| / 2 \sigma_{p}^{2}\right)$ and $R_{p}\left(X_{l}, X_{k}\right)=1$;

- if $d_{p}\left(X_{k}, X_{l}\right) \geq 0$, then $R_{p}\left(X_{k}, X_{l}\right)=1$ and $R_{p}\left(X_{l}, X_{k}\right)=\exp \left(-\left|d_{p}\left(X_{k}, X_{l}\right)\right| / 2 \sigma_{p}^{2}\right)$,

where $\sigma_{p}$ is the distance between the origin and the inflexion point of the preference function.
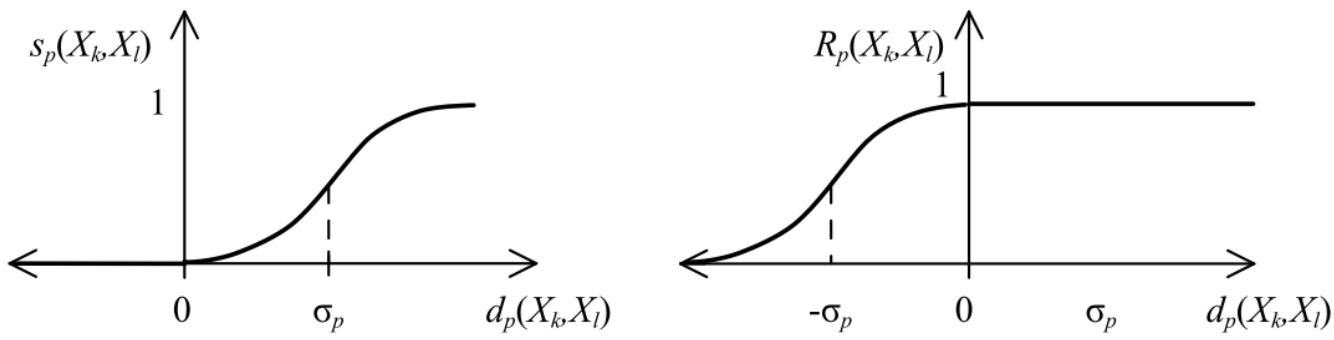

Figure 6 - Left side: Preference function based on the Gaussian criterion; Right side: NRFPR based on the Gaussian criterion, expressed in function of the difference $d_{p}\left(X_{k}, X_{l}\right)$. 
It is not difficult to demonstrate that NRFPRs constructed coherently with those six types of preference functions always satisfy weak transitivity (refer to expression (9)). For this demonstration, it is worth noting that $R_{p}\left(X_{k}, X_{l}\right) \geq R_{p}\left(X_{l}, X_{k}\right)$ only if $s_{p}\left(X_{k}, X_{l}\right) \geq 0$ (which the reader can easily verify for each type of preference function) and that a nondecreasing preference function satisfies $s_{p}\left(X_{k}, X_{l}\right) \geq 0$ only if $d_{p}\left(X_{k}, X_{l}\right) \geq 0$. In this way, if $R_{p}\left(X_{k}, X_{j}\right) \geq R_{p}\left(X_{j}, X_{k}\right)$ and $R_{p}\left(X_{j}, X_{l}\right) \geq R_{p}\left(X_{l}, X_{j}\right)$, then $d_{p}\left(X_{k}, X_{j}\right) \geq 0$ (which means that $f_{p}\left(X_{k}\right)>f_{p}\left(X_{j}\right)$ ) and, at the same time, $d_{p}\left(X_{j}, X_{l}\right) \geq 0$ (which means that $\left.f_{p}\left(X_{j}\right)>f_{p}\left(X_{l}\right)\right)$. It is also possible to infer that $f_{p}\left(X_{k}\right)>f_{p}\left(X_{j}\right)>f_{p}\left(X_{l}\right)$, which results in $d_{p}\left(X_{k}, X_{l}\right) \geq 0, s_{p}\left(X_{k}, X_{l}\right) \geq 0$, and $R_{p}\left(X_{k}, X_{l}\right)>R_{p}\left(X_{l}, X_{k}\right)$.

\section{A METHOD FOR MULTICRITERIA DECISION MAKING BASED ON THE ORLOVSKY CHOICE PROCEDURE}

The Orlovsky choice procedure makes use of a fuzzy strict preference relation given by (7) to carry out the choice of alternatives (Orlovsky, 1978, 1981). Considering the $p$ th criterion, as $P_{p}\left(X_{l}, X_{k}\right)$ describes the set of all alternatives $X_{k}$ that are strictly dominated by $X_{l}$, its compliment $\overline{P_{p}\left(X_{l}, X_{k}\right)}$ corresponds to the set of alternatives that are not dominated by $X_{l}$. Therefore, in order to meet the set of alternatives from $X$ that are not dominated by any other alternative, it suffices to obtain for each alternative $X_{k}$ belonging to $X$ the intersection of $\overline{P_{p}\left(X_{l}, X_{k}\right)}$ for all $X_{l}$ belonging to $X$. This intersection is the set of nondominated alternatives with the membership function

$$
N D_{p}\left(X_{k}\right)=\inf _{X_{l} \in X}\left\{1-P_{p}\left(X_{l}, X_{k}\right)\right\}=1-\sup _{X_{l} \in X} P_{p}\left(X_{l}, X_{k}\right),
$$

which reflects the level of nondominance of each alternative $X_{k}$.

A natural choice for a monocriteria problem based on this model should be the alternatives providing:

$$
X_{R_{p}}^{N D}=\left\{X_{k}^{N D} \in X \mid R_{p}^{N D}\left(X_{k}^{N D}\right)=\sup _{X_{k} \in X} N D_{p}\left(X_{k}\right)\right\}
$$

It is worth emphasizing that the alternatives satisfying

$$
X_{R_{p}}^{N D}=\left\{X_{k}^{N D} \in X \mid R_{p}^{N D}\left(X_{k}^{N D}\right)=1\right\}
$$

are actually nonfuzzy nondominated and can be considered as the nonfuzzy solution for the choice problem (Orlovsky, 1978, 1981).

Expressions (7), (14), and (15) may be used to solve choice or ranking problems not only with a single criterion, but also with multiple criteria. In particular, several procedures that allow one to include multiple criteria in the analysis of a decision making problem are discussed in Orlovsky (1981), Ekel \& Schuffner Neto (2006). Let us consider one of them.

Having at hand nonstrict preference relations for each criterion, one possible procedure for solving multicriteria problems consists in obtaining a global relation through the intersection of those relations as follows:

$$
G=R_{1} \cap \ldots \cap R_{q}
$$


The use of intersection to aggregate all criteria is suitable, when it is a necessary condition that a good alternative $X_{k}$ must simultaneously satisfy $F_{1}$ and $F_{2}$ and $\ldots$ and $F_{q}$. Among the $t$-norm operators, the use of the min operator, as proposed in (Orlovsky, 1981), allows one to construct the global fuzzy nonstrict preference relation

$$
G\left(X_{k}, X_{l}\right)=\min \left\{R_{1}\left(X_{k}, X_{l}\right), \ldots, R_{q}\left(X_{k}, X_{l}\right)\right\}
$$

under a completely non-compensatory approach for multicriteria decision-making, in the sense that the high satisfaction of some criteria does not relieve the remaining ones from the requirement of being satisfied (there is no compensation among the criteria). Such approach is also considered pessimistic, since it gives emphasis to the worst evaluations of each alternative.

Having at hand the global fuzzy nonstrict preference relation, equations (7), (14) and (15) can be subsequently applied directly to $G$ and the result corresponds to a fuzzy set of nondominated alternatives fulfilling the role of a Pareto set (Orlovsky, 1981).

However, in case of not being possible to distinguish two or more alternatives, the contraction of (18) is possible by differentiating the importance of $R_{p}, p=1, \ldots, q$ with the use of the weighted sum:

$$
T\left(X_{k}, X_{l}\right)=\sum_{p=1}^{q} \lambda_{p} R_{p}\left(X_{k}, X_{l}\right),
$$

where $\lambda_{p}, p=1, \ldots, q$ are importance factors of the corresponding criteria, defined as $w_{p} \in$ $[0,1]$ and $\sum_{p=1}^{q} w_{p}=1$.

The construction of $T\left(X_{k}, X_{l}\right)$, allows one to obtain the membership function $N D T\left(X_{k}\right)$ of the fuzzy set of nondominated alternatives by subsequently applying (7) and (14) to (19). The intersection

$$
Q\left(X_{k}\right)=\min \left(N D\left(X_{k}\right), N D T\left(X_{k}\right)\right),
$$

provides us with a set of alternatives with the highest level of nondominance

$$
X^{N D}=\left\{X_{k}^{N D} \mid X_{k}^{N D} \in X, Q\left(X_{k}^{N D}\right)=\sup _{X_{k} \in K} Q\left(X_{k}\right)\right\} .
$$

\section{APPLICATION EXAMPLE}

The multicriteria decision-making problem related to the site selection for constructing a new hospital is studied in Vahidnia et al. (2009). Here, we consider a simplified version of this problem, in which six sites are to be ranked, taking into account the following four criteria:

1. Distance from the arterial routes (minimization criterion);

2. Land cost (minimization criterion);

3. Population density (maximization criterion);

4. Average travel time to the nearest existing hospital (maximization criterion). 
Hospitals should be located close to main public transport routes. Here, criterion $F_{1}$ allows one to evaluate the distance from each site to the closest arterial route. Besides, for social convenience, it is important to guarantee that every person lives in an area with accessible hospital service. Criterion $F_{4}$ evaluates the average time one would take to travel between the site and the nearest existing hospital. The fifth criterion which could be considered is associated with the pollution level present at each site. However, as the sites considered here do not significantly differ with respect to this criterion, the DM decided to eliminate it from the subsequent multicriteria analysis. Figure 7 presents the fuzzy scores utilized for evaluating the third criterion, $F_{3}$-population density. Table 2 shows the evaluation matrix of the alternatives.

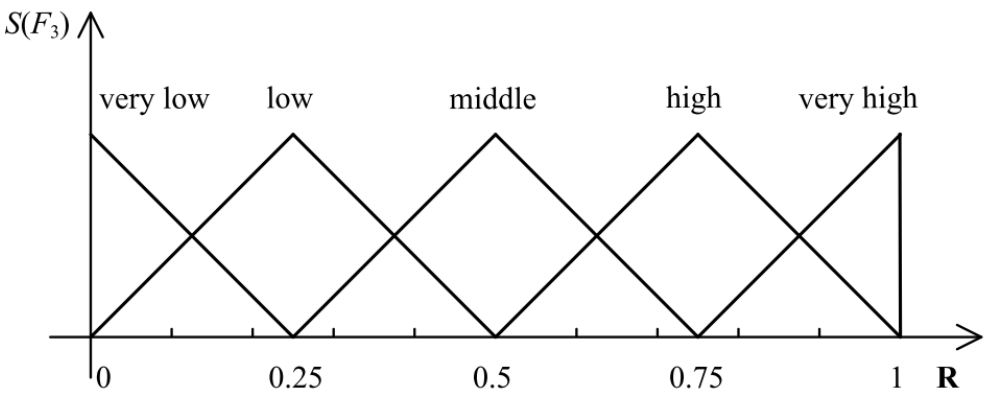

Figure 7 - Valuation of criterion $F_{3}$ (population density).

Table 2 - Evaluation matrix of the alternatives.

\begin{tabular}{|c|c|c|c|c|}
\hline & $\begin{array}{c}F_{1}\left(X_{k}\right) \\
(m)\end{array}$ & $\begin{array}{c}F_{2}\left(X_{k}\right) \\
\left(\$ / m^{2}\right)\end{array}$ & $F_{3}\left(X_{k}\right)$ & $\begin{array}{c}F_{4}\left(X_{k}\right) \\
\text { (minutes) }\end{array}$ \\
\hline$X_{1}$ & 0 & 28 & middle & 22 \\
$X_{2}$ & 350 & 24 & high & 17 \\
$X_{3}$ & 150 & 18 & high & 12 \\
$X_{4}$ & 500 & 15 & middle & 10 \\
$X_{5}$ & 50 & 8 & low & 7 \\
$X_{6}$ & 300 & 10 & very high & 5 \\
\hline
\end{tabular}

When the criterion $F_{1}$ was considered by the DM, he decided not to infer the degrees of his preferences directly from those distances listed in Table 2. The DM felt more comfortable with utilizing the multiplicative preference relation to articulate his preferences as follows:

$$
M_{1}=\left[\begin{array}{cccccc}
1 & 4 & 4 & 4 & 3 & 4 \\
1 / 4 & 1 & 1 / 3 & 3 & 4 & 1 \\
1 / 4 & 3 & 1 & 4 & 1 / 3 & 1 / 2 \\
1 / 4 & 1 / 3 & 1 / 4 & 1 & 1 / 4 & 1 / 2 \\
1 / 3 & 1 / 4 & 3 & 4 & 1 & 4 \\
1 / 4 & 1 & 2 & 2 & 1 / 4 & 1
\end{array}\right]
$$

Indeed, it is worth noting in (22) that the degrees of preference of the DM can not be directly inferred from the distances from arterial streets. For instance, although the distance of $X_{4}$ is ten 
times larger than the distance of $X_{5}, X_{5}$ is not considered ten times better than $X_{4}$. At the same time, the distance of $X_{2}$ is higher than the distance of $X_{6}$. However, the DM judged $X_{2}$ and $X_{6}$ as being indifferent to each other. Besides, it is important to indicate that although (22) does not satisfy the multiplicative transitivity, the NRFPR

$$
R_{1}=\left[\begin{array}{cccccc}
1 & 1 & 1 & 1 & 1 & 1 \\
0.226 & 1 & 0.333 & 1 & 1 & 1 \\
0.226 & 1 & 1 & 1 & 0.333 & 0.52 \\
0.226 & 0.333 & 0.226 & 1 & 0.226 & 0.52 \\
0.333 & 0.226 & 1 & 1 & 1 & 1 \\
0.226 & 1 & 1 & 1 & 0.307 & 1
\end{array}\right]
$$

constructed with the use of $H_{3}$ (refer to Table 1) satisfies the weak transitivity and this is a satisfactory level of consistency for the application of the decision-making method to be used.

In the case of criterion $F_{2}$, the DM decided to use a preference function corresponding to a Linear criterion with $b_{2}=5\left(\$ / m^{2}\right)$. The obtained NRFPR is as follows:

$$
R_{2}=\left[\begin{array}{cccccc}
1 & 0.2 & 1 & 1 & 1 & 1 \\
1 & 1 & 1 & 1 & 1 & 1 \\
1 & 1 & 1 & 0.4 & 1 & 1 \\
1 & 1 & 1 & 1 & 1 & 1 \\
1 & 1 & 1 & 1 & 1 & 1 \\
1 & 1 & 1 & 1 & 1 & 1
\end{array}\right]
$$

The population density of each alternative, which is related to criterion $F_{3}$, was estimated by him, by the linguistic terms shown in Table 2. By applying the expressions (11) and (12) to construct a NRFPR from the comparison of fuzzy estimates, the following NRFPR is obtained:

$$
R_{3}=\left[\begin{array}{cccccc}
1 & 0.5 & 0.5 & 1 & 1 & 0 \\
1 & 1 & 1 & 1 & 1 & 0.5 \\
1 & 1 & 1 & 1 & 1 & 0.5 \\
1 & 0.5 & 0.5 & 1 & 1 & 0 \\
0.5 & 0 & 0 & 0.5 & 1 & 0 \\
1 & 1 & 1 & 1 & 1 & 1
\end{array}\right]
$$

Finally, in the case of criterion $F_{4}$, a preference relation corresponding to a Quasi-criterion, with $a_{4}=3 \mathrm{~min}$. The obtained NRFPR is as follows:

$$
R_{4}=\left[\begin{array}{llllll}
1 & 1 & 1 & 1 & 1 & 1 \\
0 & 1 & 1 & 1 & 1 & 1 \\
0 & 0 & 1 & 1 & 1 & 1 \\
0 & 0 & 1 & 1 & 1 & 1 \\
0 & 0 & 0 & 1 & 1 & 1 \\
0 & 0 & 0 & 0 & 1 & 1
\end{array}\right]
$$


Having at hand the NRFPRs (23)-(26), the multicriteria analysis begins by applying (18) to obtain a global relation under a pessimistic approach through the intersection:

$$
G=\left[\begin{array}{cccccc}
1 & 0.2 & 0 & 1 & 0 & 0 \\
0 & 1 & 0 & 1 & 0 & 0 \\
0 & 0 & 1 & 0.4 & 0 & 0 \\
0 & 0 & 0.226 & 1 & 0 & 0 \\
0 & 0 & 0 & 0.5 & 0 & 0 \\
0 & 0 & 0 & 0 & 0.307 & 0
\end{array}\right]
$$

Afterward, expression (7) provides the strict preference relation

$$
P=\left[\begin{array}{cccccc}
0 & 0.2 & 0 & 1 & 0 & 0 \\
0 & 0 & 0 & 1 & 0 & 0 \\
0 & 0 & 0 & 0.174 & 0 & 0 \\
0 & 0 & 0 & 0 & 0 & 0 \\
0 & 0 & 0 & 0.5 & 0 & 0 \\
0 & 0 & 0 & 0 & 0.307 & 0
\end{array}\right]
$$

Finally, with the use of (14), we obtain the nondominance degrees of each alternative

$$
N D=\left[\begin{array}{lllllll}
1 & 0.8 & 1 & 0 & 0.693 & 1
\end{array}\right] \text {. }
$$

We can order them from best to worst as $\left(X_{1} \approx X_{3} \approx X_{6}\right) \succ X_{2} \succ X_{5} \succ X_{4}$. In order to distinguish alternatives $X_{1}, X_{3}$, and $X_{6}$, a subsequent analysis is performed by applying (19) and (20). Considering that the DM defined the weights of the criteria as $\lambda_{1}=\lambda_{2}=0.2$ and $\lambda_{3}=\lambda_{4}=0.3$, the convolution (19) provides the following matrix of pairwise comparisons:

$$
T=\left[\begin{array}{cccccc}
1 & 00.69 & 0.65 & 0.8 & 0.8 & 0.55 \\
0.545 & 1 & 0.667 & 0.8 & 0.8 & 0.65 \\
0.545 & 0.7 & 1 & 0.88 & 0.667 & 0.554 \\
0.545 & 0.417 & 0.695 & 1 & 0.645 & 0.404 \\
0.417 & 0.245 & 0.4 & 0.85 & 1 & 0.7 \\
0.545 & 0.7 & 0.7 & 0.7 & 0.781 & 1
\end{array}\right]
$$

In this way, by processing (30) with the use of (7) and (14), it is possible to obtain the following fuzzy set of nondominated alternatives:

$$
N D T=\left[\begin{array}{llllll}
0.95 & 0.855 & 0.854 & 0.617 & 0.445 & 1
\end{array}\right]
$$

Finally, the intersection of (29) and (30), obtained in accordance with (20), makes it possible to contract the fuzzy set of nondominated alternatives as follows:

$$
N D=\left[\begin{array}{llllll}
0.95 & 0.8 & 0.854 & 0 & 0.445 & 1
\end{array}\right] \text {. }
$$

The final ranking of the alternatives can be defined on the basis of (32) as

$$
X_{6} \succ X_{1} \succ X_{3} \succ X_{2} \succ X_{5} \succ X_{4} .
$$




\section{CONCLUSIONS}

In this paper we showed how the preference functions can be utilized to construct NRFPRs. The preference functions correspond to a well established preference format which has been utilized to model the preferences of a DM in the methods PROMETHEE I and PROMETHEE II. The results of this paper allow a more flexible input of preferences in the decision-making process based on the analysis of $\langle X, R\rangle$ models (now, it is possible to offer to a DM the following formats: the ordering of the alternatives, the utility values, the multiplicative preference relations, the fuzzy estimates, the reciprocal as well as the nonreciprocal fuzzy preference relations and the preference functions). The applicability of the proposed procedure is demonstrated through the solution of a multicriteria decision-making problem related to the site selection for constructing a new hospital.

\section{ACKNOWLEDGMENTS}

This research is supported by the National Council for Scientific and Technological Development of Brazil (CNPq) - grants PQ: 307406/2008-3 and PQ: 307474/2008-9.

\section{REFERENCES}

[1] Alsina C. 1985. On a family of connectives for fuzzy sets. Fuzzy Sets and Systems, 16: 231-235.

[2] BeLton V. 1999. Multiple-criteria problem structuring and analysis in a value theory framework. In: Multicriteria decision making: advances in MCDM models, algorithms, theory [edited by GAL T, Stewart T \& Hanne T], Kluwer Academic Publishers, Dordrecht, 12-2-12-29.

[3] BRANS JP \& VincKe PH. 1985. A preference ranking organization method (the PROMETHEE method for multiple criteria decision-making. Management Science, 31: 647-656.

[4] De BAETs B \& Fodor JC. 1997. Twenty years of fuzzy preference structures. Rivista di Matematica per le Scienze Economiche e Sociali, 20: 45-66.

[5] Chiclana F, Herrera F \& Herrera-Viedma E. 1998. Integrating three representation models in fuzzy multipurpose decision making based on fuzzy preference relations. Fuzzy Sets and Systems, 97: $33-48$.

[6] Chiclana F, Herrera F \& Herrera-Viedma E. 2001. Integrating multiplicative preference relations rain a multipurpose decision-making model based on fuzzy preference relations. Fuzzy Sets and Systems, 122: 277-291.

[7] EKel P, PEDRYCZ W \& SCHINZINGER R. 1998. A general approach to solving a wide class of fuzzy optimization problems. Fuzzy Sets and Systems, 97: 49-66.

[8] Ekel P.YA. \& Schuffner Neto FH. 2006. Algorithms of discrete optimization and their application to problems with fuzzy coefficients. Information Sciences, 176: 2846-2868.

[9] Farquhar PH \& Keller LR. 1989. Preference intensity measurement. Annals of Operations Research, 19: 205-217.

[10] Fodor JC \& Roubens M. 1994a. Fuzzy preference modelling and multicriteria decision support, Kluwer, Boston. 
[11] Fodor JC \& Roubens M. 1994b. Valued preference structures. European Journal of Operational Research, 79: 277-286.

[12] Herrera-Viedma E, Herrera F \& Chiclana F. 2002. A consensus model for multiperson decision making with different preference structures. IEEE Transactions on Systems, Man and Cybernetics - Part A: Systems and Humans, 32: 394-402.

[13] Herrera-Viedma E, Herrera F, Chiclana F \& Luque M. 2004. Some issues on consistency of fuzzy preference relations. European Journal of Operational Research, 154: 98-109.

[14] Kulshreshtha P \& SheKar B. 2000. Interrelations among fuzzy preference-based choice functions and significance of rationality conditions: A taxonomic and intuitive perspective. Fuzzy Sets and Systems, 109: 429-425.

[15] Lu J, Zhang G, RuAn D \& Wu F. 2007. Multi-objective Group Decision Making: Methods, Software and Applications with Fuzzy Set Techniques. Imperial College Press, London.

[16] ORLOVSKY SA. 1978. Decision-making with a fuzzy preference relation. Fuzzy Sets and Systems, 1: $155-167$.

[17] ORLovsky SA. 1981. Problems of Decision Making with Fuzzy Information (in Russian), Nauka, Moscow.

[18] Pedrycz W, Ekel P \& Parreiras R. 2011. Fuzzy Multicriteria Decision-Making: Models, Methods, and Applications. Wiley, Chichester.

[19] QueIroz JCB. 2009. Models and Methods of Decision Making Support for Strategic Management: $\mathrm{PhD}$ thesis (in Portuguese), Federal University of Minas Gerais, Belo Horizonte.

[20] SaAty T. 1980. The Analytic Hierarchy Process. McGraw Hill, New York.

[21] Sengupta K. 1998. Fuzzy preference and Orlovsky choice procedure. Fuzzy Sets and Systems, 93: 231-234.

[22] Vahidnia MH, Alesheikh AA \& Alimohammadi A. 2009. Hospital site selection using fuzzy AHP and its derivatives. Journal of Environmental Management, 90: 3048-3056.

[23] WANG X. 1997. An investigation into relations between some transitivity-related concepts. Fuzzy Sets and Systems, 89: 257-262.

[24] Zhang Q, WANG Y \& YANG Y. 2007. Fuzzy multiple attribute decision making with eight types of preference information on alternatives. In: Proc. of the 2007 IEEE Symposium on Computational Intelligence in Multicriteria Decision Making, Honolulu, 288-293. 